\title{
DEUTSCHE LITERATURZEITUNG
}

HERAUSGEBER: WERNER HARTKE

Redaktionsausschuß:

W. Bahner · H. Bertsch · M. Buhr · W. Hartke · H. Kraatz - W. Lange · H.-J. Treder · E. Winter

I N HALT

Geschichte und Organisation der Wissenschaft

Antonius Gvilielmus Amo Aferaus Axim in G h a n a. Dokumente, Autographe, Belege. Ausgew. u. zsgest. v. B. Brentjes. - Antonius Gvilielmus Amo Afer of A xim in G h a n a. Transl. of his Works. Ed. D. SiegSpalte 529

М. Т. Белявский, В. В. Сорокин, Памятные места Старого здания Московского университета. [M. T. Beljavskij, v. v. Sorokin, Die Erinnerungsstătten des Alten Gebăudes der Moskauer Universität.] (Conrad Grau, Dr. phil. habil., Berlin.)

V a dim Nikolajew, Forschung und Entwicklung im Imperialismus. (Wolfgang Schlicker, Dr. phil., Potsdam.)

\section{Philosophie}

L u ci e n S è v e, Marxismus und Theorie đer Persönlichkeit. Aus d. Franz. (Ileana Bauer, Dr. phil., Berlin.) . Spalte 534 P. C. Ка риин ская, Фплософскше проблемы молекулярной биологии. [R. S. Karpinskaja, Philosophische Probleme der Molekularbiologie.] (Karl-Hermann Köhler, Dr. sc. rer. nat., Greifswald.) . . . . . . . . . . Spalte 537 M a r i o B u n ge, Philosophy of Physics. (Martin Strauss, Gottlob Frege, Conceptual Notation and Related Articles. Transl and ed. with a Biography and Introduction by T. W. Bynum. (Helmut Metzler, Dr. phil., Jena.) Spalte 542 Lothar Krauth, Die Philosophie Carnaps. (Wolfram L o th a r $\mathrm{Kr}$ a $\mathrm{u} \mathrm{th}$, Die Philosophie Carnaps.
Heitsch, Dolfram
Spalte 544

\section{Allgemeine Sprachwissenschaft}

Sprache und Ideologie. Beitrăge zu einer marx.leninist. Sprachwirkungsforschung. Hrsg. v. W. Schmidt. (ErC. K. II аумян, Философские вопросы теоретической лингвистики. [S. K. Saumjan, Philosophische Fragen der theoreti-

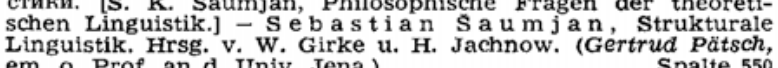
em. o. Prof. an d. Univ. Jena.) . . . . . Spalte 550 Fragen der strukturellen syntax und der kontrastiven Grammatik. Mit Beiträgen von 0 . Leys, J. Lerot, W. Abraham u. a. (Reinhard Sternemann, Dr.
phil. habil., Berlin.). Spalte 552

\section{Klassische und mittellateinische Philologie}

T $11 \mathrm{man} \mathrm{Kr}$ ischer, Formale Konventionen der homerischen Epik. (Rismag Gordesiani, Dr. phil., Tbilissi.) A n n e L e b e c k, The Oresteia. (Dietrich Ebener, Prof. Dr.
phil. habil., Potsdam-Rehbrucke.) И. Н. Голепи щев-К у у зов, Средневековая латинская литеparypa Италии. [I. N. Golenišcev-Kutuzov, Die mittelalterliche
lateinische Literatur Italiens.] (Judith Matveevna Kagan, Dr. lateinische Literatur Italiens.] (Judith Matveevna Kagan, Dr.
phil., Moskva.).

\section{Romanistik}

L ew S. Gordon, Studien zur plebejisch-demokratischen Tradition in der französischen Aufklärung. Aus d. Russ. (Rolf Geißler, Dr. phil., Berlin.). . . . . . Spalte 563 Recherches nouvelles sur quelques écri$\mathrm{v}$ a i n $\mathrm{s}$ des $1 \mathrm{um}$ ièr es. Sous la direction de J. Proust. (Martin Fontius, Dr. phil., Berlin.) . . . Spalte 566 $\mathrm{H}$ a ns-G ünter Funke, Crébillon fls als Moralist und Gesellschaftskritiker. (Werner Krauss, em. Prof. Dr. phil.,

\section{Anglistik}

English Literature $1660-1800$. Founded by R. S. Crane. Vol. V: 1961-1965; Vol. VI: 1966-1970. (Fritz Rau, Dr.
phil., Leverkusen.) Patricia M. B a ll, The Science of Aspects. The Changing Role of Fact in the Work of Coleridge, Ruskin, and Hop kins. (Horst Höhne, Dr. phil., Rostock.). .'. Spalte 572

\section{Germanistik}

Si ebenbürgisch-s ächsisches worterbuch In Verb. mit d. Dt. Akad. d. Wiss. zu Berlin hrsg. von d. Akad. d. Sozialistischen Republik Rumänien. Bd 3: G. (Heinz Sperschneider, Dr. phil., Jena.). Spaite 575 Philipp von Zesen 1619-1969. Hrsg. v. F. v. Ingen. (Eberhard Haufe, Dr. phil., Weimar.).' Spalte 578 Marlies P r üsen er, Lesegesellschaften im achtzehnten Jahrhundert. (Othmar Feyl, Prof. Dr. phil. habil., Berlin.)

E rha rd B a hr, Die Ironie im Spätwerk Goethes - ... diese sehr ernsten Scherze...". (Hans Jürgen Geerdts, 0 Peter von Matt, Die Augen der Automaten. E. T. A Peter von Mat t, Die Augen der Automaten. E. T. A. Hoffmanns Imaginationslehre als Prinzip seiner Erzăhlkunst.
(Hans-Georg Werner, o. Prof. an d. Univ. Halle.) Spalte 585 O livier B o e c k, Heines Nachwirkung und Heine Parallelen in der französischen Dichtung. (Fritz Mende, Dr. paed. Weimar.) .. . . . . . . . . . . Spalte 588 Der Briefwechsel zwischen Theodor Font a n e u n d P P u l He y s e. Hrsg. v. G. Erler. (Joachim W $11 \mathrm{~h}$ e $1 \mathrm{~m}$ R a a b e. Briefwechsel Raabe - Jensen. Bearb. v. W $11 \mathrm{~h} \mathrm{e} 1 \mathrm{~m}$ R a a b e. Briefwechsel Raabe - Jensen. Bearb. v. E. Hoppe u. H. Oppermann. (Joachim Muller, em. O. Prot. an
d. Univ. Jena.) . . . . . . . Spalte 593 Slawistik

Ii. C. Горбачевич, Измешение норм русского литературного языка. [K. S. Gorbacevic, Der Wandel der Normen in der russischen Literatursprache.] (Klaus Müller, Dr. phil. habil., Spalte 597

A. Х ватов, Худонественнй мир Шолохова. [A. Chvatov, Die künstlerische Welt Scholochows.] (Barbara Hiller, Dr. phil.,

\section{Baltistik}

A lexander Kursch at, Litauisch-Deutsches Wörterbuch. Hrsg. v. W. Wissmann + u. E. Hofmann. (Rainer Eckert,
Dr. phil., Leipzig.)

\section{Orientalistik}

Hans L. Gottschalk, Bertold Spuler, Hans $\mathrm{K}$ ähler, Die Kultur des Islams. (Manfred Fleischhammer, o. Prof. an d. Univ. Halle.) D e r I s I a m. Bd II: Die islamischen Reiche nach dem Fall von Konstantinopel. Hrsg. v. G. E. v. Grunebaum. - E r n s $\mathrm{K}$ ü h n e 1, Islamische Schriftkunst. 2. Aufl. (Burchard Brentjes, Prof. Dr. phil. habil., Berlin.). . . . . . Spalte 606 W a 1 ther H issig, Geschichte der mongolischen Literatur. Bd I u. II. (Siegfried Behrsing, o. Prop. an d. Univ. Berlin.)

\section{Alte Geschichte}

Spalte 608

$\mathrm{J}$ o s e ph Vog t, Sklaverei und Humanität. 2., erw. Aufl (Liselot Huchthausen, Dr. sc. phil., Rostock.). '. Spalte 61 (Fortsetzung des Inhalts auf Umschlagseite 3.)

Die Deutsche Literaturzeitung erscheint im Akademie-Verlag, 108 Berlin, Leipziger Straße $3 / 4$ Redaktion: 104 Berlin, Schiffbauerdamm 19 (Akademie der Wissenschaften der DDR)

Manuskriptsendungen sind sämtlich an die Adresse der Redaktion zu richten. - Wir bitten, Rezensionsexemplare im Interesse schneller Berichterstattung an die Redaktion zu senden. - Nachdruck nur mit Genehmigung der Redaktion gestattet. Mitteilungen geschäftlichen Charakters sind sämtlich an den Verlag zu richten. Die Deutsche Literaturzeitung erscheint monatlich. Der Bezugspreis beträgt vierteljährlich M 12,- (Sonderpreis für die DDR M 6,-), Einzelheft M 4,- (Sonderpreis für die DDR M 2,-) und Bestellgebuhr. Bezugspreis dieses Doppelheftes M 8,- (Sonderpreis für die DDR M 4,-). Abbestellungen können nur bis vler Wochen vor Quartalsende anerkannt werden, andernfalls wird das folgende Quartal noch geliefert. Printed in the German Democratic Republic. 